\title{
O033: Is MRSA inpatient transmission driving high MRSA hospital importation in the US veterans affairs?
}

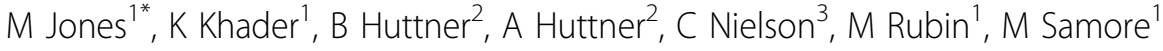 \\ From 2nd International Conference on Prevention and Infection Control (ICPIC 2013) \\ Geneva, Switzerland. 25-28 June 2013
}

\section{Introduction}

The prevalence of methicillin-resistant Staphylococcus aureus (MRSA)-carriage among hospital admissions (importation) is high in United States Veterans Affairs (VA) hospitals when compared to published studies from other settings. We investigated whether inpatient transmission alone could explain this finding.

\section{Methods}

We used retrospective clinical data from 112 VA hospitals to calibrate a stochastic compartmental simulation model. We modeled a typical VA hospital with 70 beds and its surrounding community with 38,000 enrolled Veterans. The model consisted of 6 patient states-susceptible and colonized individuals who had never been hospitalized, were currently hospitalized, or had a history of hospitalization. Admission, length of stay, inpatient acquisition rates, and mortality rates were calibrated to observed data for MRSA-positive and negative populations. The relative rate of admission of MRSA-positive to negative populations was set to 1.2, based on the relative rate of readmission. Importation among first time admissions was 6.8\%; overall it was $10.5 \%$. Readmission rates were calibrated to reflect $15 \% 30$ day readmission[1]. We compared a base-case scenario assuming $5 \%$ assumed MRSA prevalence among new Veterans entering the population and a scenario without inpatient transmission. Each scenario was run 200 times.

\section{Results}

In the base-case scenario, the median importation was 10.2\% (IQR 9.7-10.8\%), the median outpatient prevalence was $5.0 \%$, and the median outpatient acquisition rate was $1.5 / 100,000$ person-years. Without inpatient transmission, the median importation dropped to $3.5 \%$ (IQR 3.3$3.6 \%)$.

\section{Conclusion}

Dynamic theory predicts that discharge prevalence, outpatient transmission, and readmission rates influence importation. This modeling study demonstrated a large impact of inpatient transmission on MRSA importation prevalence when other factors were held constant. It appears plausible that the high importation prevalence observed in VA hospitals may be attributed to the nosocomial acquisition and readmission of prevalent patients. A sensitivity analysis is necessary to assess the robustness of this finding to our assumptions.

\section{Disclosure of interest}

None declared.

\section{Author details}

${ }^{1}$ Epidemiology, VA Salt Lake City HCS, Salt Lake City, USA. ${ }^{2}$ Infection Control Program, Geneva University Hospitals and Faculty of Medicine, Geneva, Switzerland. ${ }^{3}$ Veterans Affairs Reno Medical Center, Reno, USA.

Published: 20 June 2013

\section{Reference}

1. Ann Intern Med 2012, 157(12):837-845.

doi:10.1186/2047-2994-2-S1-O33

Cite this article as: Jones et al:: O033: Is MRSA inpatient transmission driving high MRSA hospital importation in the US veterans affairs? Antimicrobial Resistance and Infection Control 2013 2(Suppl 1):033.

${ }^{1}$ Epidemiology, VA Salt Lake City HCS, Salt Lake City, USA

Full list of author information is available at the end of the article

(c) 2013 Jones et al; licensee BioMed Central Ltd. This is an Open Access article distributed under the terms of the Creative Commons 